\title{
The variable link between PNA and NAO in observations and in multi-century CGCM simulations
}

\author{
Joaquim G. Pinto $\cdot$ Mark Reyers $\cdot$ Uwe Ulbrich
}

Received: 20 May 2009/Accepted: 12 February 2010/Published online: 6 March 2010

(c) The Author(s) 2010. This article is published with open access at Springerlink.com

\begin{abstract}
The link between the Pacific/North American pattern (PNA) and the North Atlantic Oscillation (NAO) is investigated in reanalysis data (NCEP, ERA40) and multicentury CGCM runs for present day climate using three versions of the ECHAM model. PNA and NAO patterns and indices are determined via rotated principal component analysis on monthly mean $500 \mathrm{hPa}$ geopotential height fields using the varimax criteria. On average, the multicentury CGCM simulations show a significant anti-correlation between PNA and NAO. Further, multi-decadal periods with significantly enhanced (high anti-correlation, active phase) or weakened (low correlations, inactive phase) coupling are found in all CGCMs. In the simulated active phases, the storm track activity near Newfoundland has a stronger link with the PNA variability than during the inactive phases. On average, the reanalysis datasets show no significant anti-correlation between PNA and NAO indices, but during the sub-period 1973-1994 a significant anti-correlation is detected, suggesting that the present climate could correspond to an inactive period as detected in the CGCMs. An analysis of possible physical mechanisms suggests that the link between the patterns is established by the baroclinic waves forming the North Atlantic
\end{abstract}

\section{J. G. Pinto $(\bowtie) \cdot$ M. Reyers}

Institute for Geophysics and Meteorology,

University of Cologne, Kerpener Str. 13,

50923 Cologne, Germany

e-mail: jpinto@meteo.uni-koeln.de

M. Reyers

e-mail: mreyers@meteo.uni-koeln.de

\section{U. Ulbrich}

Institute for Meteorology, Freie Universität Berlin, Carl-Heinrich-Becker-Weg 6-10, 12165 Berlin, Germany

e-mail: ulbrich@met.fu-berlin.de storm track. The geopotential height anomalies associated with negative PNA phases induce an increased advection of warm and moist air from the Gulf of Mexico and cold air from Canada. Both types of advection contribute to increase baroclinicity over eastern North America and also to increase the low level latent heat content of the warm air masses. Thus, growth conditions for eddies at the entrance of the North Atlantic storm track are enhanced. Considering the average temporal development during winter for the CGCM, results show an enhanced Newfoundland storm track maximum in the early winter for negative PNA, followed by a downstream enhancement of the Atlantic storm track in the subsequent months. In active (passive) phases, this seasonal development is enhanced (suppressed). As the storm track over the central and eastern Atlantic is closely related to the NAO variability, this development can be explained by the shift of the NAO index to more positive values.

Keywords PNA - NAO - Decadal variability · Storm track $\cdot$ CGCM

\section{Introduction}

The large-scale variability over the mid- and high latitudes of the Northern Hemisphere features regions which are linked via teleconnections, with their cores often being called "centres of action". Wallace and Gutzler (1981) and Barnston and Livezey (1987) identified the Pacific-North Atlantic Oscillation (PNA) and the North Atlantic Oscillation (NAO) as the two most prominent teleconnection patterns over the Northern Hemisphere (NH) extratropics. The PNA is a 3-center pattern extending over most of the North Pacific (NP) and North America (Wallace and 
Gutzler 1981), usually identified from $500 \mathrm{hPa}$ geopotential heights. It is associated with changes of synoptic activity and subtropical jet stream over the NP (e.g. Franzke et al. 2009) and affects the climate conditions over large parts of the NP and the North American Continent (e.g. Rogers and Raphael 1992; Archambault et al. 2008). The NAO is a dipolar pattern of mean sea level pressure over the North Atlantic (NA) extending from the subtropical (Azores high) to sub-Artic latitudes (Icelandic low; e.g. Hurrell 1995). It is associated with westerly winds variations over the Eastern NA and Western Europe, thus being an important factor for winter climate in Europe (e.g. Wanner et al. 2001; Hurrell et al. 2003). The NAO is associated with intensity changes and north/south displacements of the mid-latitude jet and synoptic activity over the NA (e.g. Luo et al. 2007; Pinto et al. 2009).

The discussion of possible mechanisms steering the NAO has been an issue in recent decades. Several authors have suggested that the NA SSTs force the NAO on decadal and longer time scales (e.g. Rodwell et al. 1999; Manganello 2008; see Wanner et al. 2001 for a review). The respective SST anomalies could arise from modulations of the oceanic gyre circulation (e.g. Grötzner et al. 1998) or feedback processes related to the thermohaline overturning at high latitudes (e.g. Timmermann et al. 1998). On the other hand, atmospheric influences on the ocean are also active, and a realistic NAO is produced even in coupled global circulation models (CGCMs) which do not include a dynamical ocean (Christoph et al. 2000). Other authors suggest influences from Indo-Pacific SST anomalies (e.g. Hoerling et al. 2001) and from tropical Pacific variability (particularly the ENSO phenomenon, e.g. Trenberth et al. 1998; Lin et al. 2005; Sterl et al. 2008; Müller and Roeckner 2008; Müller et al. 2008). In addition, anomalies of snow cover over Eurasia have been linked to the variability of the NAO (e.g. Saito and Cohen 2003).

A connection between the NP and NA atmospheric circulation anomalies has been considered by a number of authors. Based on data from 1899 to 1975, Van Loon and Rogers (1978) identified a negative correlation between the monthly mean pressures of the Icelandic low and the Aleutian low in winter (December-February). Bongioannini Cerlini et al. (1999) computed indices of PNA and NAO on a monthly time scale and found a correlation of -0.42 for also for boreal winter (1949-1994). Honda et al. (2001) found a negative correlation between the intensities of the Aleutian and the Icelandic lows in NCEP re-analysis (1973-1994), reaching a value of -0.7 for the period February to Mid-March (cf. also Orsolini et al. 2008). Recently, Song et al. (2009) concluded that correlations between PNA and NAO on a daily scale are largest (and statistically significant) with zero time lag and in a range of lags of \pm 10 days. Thus, there is ample evidence for the existence of such a link between the PNA and the NAO.

Several studies found that the correlation between the Pacific and Atlantic regions undergo decadal changes. With respect to storm tracks, Chang (2004) found a significant positive link between the regions of main winter activity in the period $1975 / 1976$ to $1998 / 1999$, while correlations were low in an earlier period (1957/1958-1971/1972). He speculated that this effect might be an artefact of low aircraft observations prior to about 1970 and thus to an error in the reanalysis datasets, as model simulations driven with observed SSTs produce a weak link even for the later period. A variable link is, however, tentatively supported by the study of Knippertz et al. (2003), who looked at the changing correlations between ENSO and the NAO. Further, Raible et al. (2001) observed two decadal regimes for the NAO-variability in ECHAM4 model simulations: (a) phases with enhanced low-frequency variability ("active") characterized by regional modes over the NA and NP (here with a barocline PNA mode) and (b) phases with reduced low-frequency variability ("inactive"), characterized by a global mode with a dominant PNA pattern and stronger links to SST anomalies over the tropical Pacific.

This statistical relation between PNA and NAO is also relevant when considering the Arctic Oscillation (AO) or Northern Annular Mode (e.g., Thompson and Wallace 1998). The AO has a similar structure to the NAO, but is zonally more symmetric (e.g. Thompson and Wallace 1998, 2000; Wallace 2000). There has been much discussion on the inter-relationships between PNA, NAO and AO and also on the influence of the stratospheric vortex variability in the troposphere (e.g. Baldwin and Dunkerton 1999; Ambaum and Hoskins 2002; Thompson et al. 2002; Scaife et al. 2005; Orsolini et al. 2008; Castanheira et al. 2009). For example, Baldwin and Dunkerton (2001) provided evidence that strength of the stratospheric vortex affects not only the phase of the AO/NAO indexes, but also the location of the mid-latitude storm tracks and the likelihood of storms. Further, Castanheira and Graf (2003) stated that the coupling between the atmospheric circulation over the NA and NP is larger in months with strong stratospheric polar vortex. Finally, Orsolini et al. (2008) have recently analyzed the formation and life cyclone of the Aleutian-Icelandic Low Seesaw in an atmospheric GCM with a well resolved stratosphere, and they identified a clear extension of this Seesaw into the stratosphere, where its presence modulates the polar night jet intensity.

Feldstein $(2002,2003)$ investigated the nature of both PNA and NAO modes and concluded that the most important difference is that while PNA life cycle is dominated by linear processes, the variability of the NAO is dominated by nonlinear processes. Further, and unlike the PNA, the NAO may be interpreted as a forced phenomenon (Feldstein 2003). As a 
consequence, one may expect that the link between PNA and NAO is produced by mechanisms modulated by PNA. Benedict et al. (2004), Franzke et al. (2004) and Rivière and Orlanski (2007) suggested that the synoptic-scale waves propagating from the eastern Pacific and subsequent Rossby wave-breaking play a dominant role in the determination of the phase of the NAO. According to Benedict et al. (2004), this is even true for the maintenance of the NAO phase by a succession of breaking of upstream synoptic scale waves. If such disturbances are no longer present, the NAO phase decays. They also pointed out to the differences between the two NAO phases: while the positive phase is characterized by two breaking waves (one over the NA, one over the North American west coast), the negative phase is characterized by a single wave-breaking over the NA. Of course, the regional relationship between synoptic waves and the NAO is twosided, as explored in a number of studies (e.g. Schneidereit et al. 2007; Pinto et al. 2009). Further, blocking also plays a role in the link between NAO and cyclones: Croci-Maspoli et al. (2007) and Woollings et al. (2008) found frequent NA blocking events in negative NAO phases, while positive phases are largely characterized by unblocked situations.

In the current paper, we consider a potential PNA influence on the NAO imposed by a modulation of growth conditions of tropospheric eddies over eastern North America and western NA. This is analysed using observational data (NCEP and ERA40 re-analysis) and multicentennial simulations with coupled Ocean-Atmosphere CGCMs. Such multi-centenial CGCMs simulations are an optimal base to analyse these relationships, as they also permit to assess possible long term (decadal, centennial) regime variations of the intensity of the coupling. We will put the focus on considerations of the troposphere as two of the three CGCMs considered do not include a well resolved stratosphere.

This paper is structured as follows: Information on the Reanalysis datasets, the coupled CGCMs and on the considered methodologies are described in Sect. 2. In Sect. 3, the connection between the PNA, the NAO and the NA storm track are analyzed, considering possible long term variations of the intensity of the coupling. Further, a physical mechanism for the modulation of eddy activity in the core of the NA storm track is proposed. A short discussion concludes this paper.

\section{Data and methods}

As observational data we use National Center for Environmental Prediction re-analyses for 1950-1999 (Kistler et al. 2001; hereafter NCEP) and the European Center of Medium Range Weather Forecast reanalysis data for 19582001 (Uppala et al. 2005; hereafter ERA40). Unless otherwise stated, we consider boreal winter means in our study, covering the period November to March.

The multi century control runs have been conducted with three different versions of the ECHAM model. One is a 300-year simulation with the ECHAM4/OPYC3 CGCM (Bacher et al. 1998; hereafter ECHAM4) in T42L19 resolution for present-day conditions. The uppermost vertical model level is at a pressure of $30 \mathrm{hPa}$. The representation of the spatial structure, the variability of the NAO and of the mid tropospheric storm tracks have been found to be realistic in previous studies (Ulbrich and Christoph 1999; Christoph et al. 2000). Ocean-Atmosphere coupling involved an annual mean flux correction, restricted to heat and freshwater fluxes (in order to avoid climate drift). The model produces a generally realistic ENSO variability (Roeckner et al. 1996).

Secondly, a pre-industrial control simulation (505 years with fixed 1860 GHG concentrations) with the CGCM ECHAM5/MPI-OM1 is considered (hereafter ECHAM5). The atmospheric model has a spatial resolution of T63 and 31 vertical levels (Roeckner et al. 2003), reaching up to $10 \mathrm{hPa}$. The model does not employ flux adjustments. Further details can be found in Roeckner et al. (2003; 2006) and Jungclaus et al. (2006). This model also produces a generally realistic ENSO variability (Jungclaus et al. 2006). The pre-industrial run was preferred to the available present day control run as the latter was much shorter (only 100 years long). Storm track and NAO variability of this pre-run have been previously considered e.g. by Bengtsson et al. (2006) and Pinto et al. (2007).

Thirdly, a 300-year present climate control simulation performed with the ECHO-G with Middle Atmosphere Model (Hübener et al. 2007; hereafter EGMAM) CGCM is analysed. The basis is the ECHO-G coupled model (ECHAM4/HOPE-G, cf. Legutke and Voss 1999), now extended into the stratosphere (Manzini and McFarlane 1998). EGMAM has 39 layers (model top: $0.01 \mathrm{hPa}$, circa $80 \mathrm{~km}$ ), and the horizontal atmospheric resolution is T30. Similarly to ECHO-G, EGMAM shows a 2-year peak on the ENSO variability (Min et al. 2005). A flux correction is applied for heat and freshwater exchange and is on global average zero. For more details see Hübener et al. (2007) and Spangehl et al. (2009).

From each dataset, $500 \mathrm{hPa}$ geopotential height is used to analyse possible anomalies in the mid-tropospheric large scale circulation. As a measure of baroclinic wave activity, we consider the storm track intensities from daily $500 \mathrm{hPa}$ data (cf. e.g. Blackmon 1976). The storm track is obtained by first applying a bandpass filter (half power cut-off periods: 2.5 and 8 days; see Christoph et al. 1995) to the daily $500 \mathrm{hPa}$ geopotential height fields at each gridpoint and subsequently computing the standard deviation. Storm track intensities for the three CGCMs and re-analyses are 
found to be in close agreement with respect to structures and intensities (cf. Appendix, Fig. 13). As a measure of barocliniticy we consider the maximum Eady growth rate (cf. Hoskins and Valdes 1990), defined as BI $=0.31$ ( $f$ $N) \times|\mathrm{d} v / \mathrm{d} z|$, where $f$ is the Coriolis parameter, $N$ is the static stability, $z$ the vertical coordinate and $v$ the horizontal wind vector. It quantifies the large-scale conditions for the potential growth of cyclones and is a good approximation of wave growth in observations even with longitudinally variable mean flow (Hoskins and Hodges 2002). We consider this quantity for the upper $(300-500 \mathrm{hPa})$ troposphere. In order to investigate the possible role of latent heat, lower tropospheric humidity at $850 \mathrm{hPa}$ is also analysed. The upper-air jet stream is considered in the upper troposphere, corresponding to the zonal wind speed at $250 \mathrm{hPa}$.

\section{Results}

\subsection{PNA, NAO and North Atlantic and North Pacific storm tracks}

The first step of our investigation is the determination of the PNA and NAO patterns as represented in the considered CGCMs and reanalysis datasets. The patterns are obtained by performing a rotated principal component analysis on $500 \mathrm{hPa}$ geopotential height fields for the $\mathrm{NH}$ north of $20^{\circ} \mathrm{N}$. This technique permits the derivation of both patterns in a consistent way for both CGCM and reanalysis data. Both the NAO and the PNA patterns are among the five leading rotated empirical orthogonal functions (REOFs). The three CGCMs produce a realistic NAO and PNA in terms of structure and explained variances in comparison to the reanalysis data (cf. Appendix, Figs. 14, 15). However, please note that the patterns are not identical, even not between ERA40 and NCEP. The latter effect may partially be attributed to the different time windows covered by these datasets. A statistically significant anticorrelation (ranging between $r=-0.22$ and $r=-0.41$,

Table 1 Characteristics of PNA and NAO indexes for the 3 CGCMs (ECHAM4, ECHAM5, EGMAM) and reanalysis (NCEP, ERA40) based on winter mean data: explained variance of the NAO pattern (NAO); explained variance of the PNA pattern (PNA); correlation
99\% significance level) is found between PNA and NAO indices for the CGCMs, while no correlation is found for both reanalysis datasets (cf. Table 1).

Next, we analyse the relationships between PNA, NAO, and the storm track over both NH oceanic basins. The NP storm track activity is related to the phase of the PNA, while the NA storm track has a relationship to both the PNA and the NAO. In order to quantify these relationships, we analyse the influence of the PNA over the $\mathrm{NH}$ in terms of its impact on $500 \mathrm{hPa}$ geopotential height fields and storm track activity. With this aim, composite fields for all years with negative PNA (hereafter PNA-) and for those with positive PNA (hereafter PNA+) are produced. The difference between both composites (PNA- minus PNA+) are shown in Fig. 1 for the three CGCMs. Results show that years of PNA - are characterized by above normal $500 \mathrm{hPa}$ geopotential height reaching from southeastern North America to the subtropical NA (around $30^{\circ}-40^{\circ} \mathrm{N}$ ), and a negative anomaly further north (around $60^{\circ} \mathrm{N}$ ). Further, enhanced storm track activity is found both over the central NP and over the western NA (cf. Fig. 1) for PNA(which is associated with a weak Aleutian trough). The corresponding explained variance for the NA storm track core over Newfoundland is as high as $30 \%$ (for ECHAM4, correlation field not shown). These results indicate that the PNA variability is associated with changes in baroclinic wave activity over the core area of the NA storm track, and that there is a poleward (equatorward) shift of storm track activity in the negative (positive) PNA phase over the NP. Note that the NA storm track anomalies for ECHAM5 do not extend as far east as the other CGCMs (cf. Fig. 1).

In terms of the observational data, PNA variability is associated with a tripole of $500 \mathrm{hPa}$ geopotential height anomalies, which closely resembles the PNA-pattern itself (Fig. 2a, c). PNA changes are significantly related to the intensity of the Newfoundland storm track maximum in the ERA40 reanalysis (Fig. 2d), while the anomaly in the NCEP reanalysis is not statistically significant (Fig. 2b).

Next, we analyse the influence of the NAO on the NA $500 \mathrm{hPa}$ storm track (computed as the composite difference

between PNA and NAO indexes (Corr); percentage of mismatches (Mis; PNA and NAO of opposite sign); percentage of months with $\mathrm{PNA}-$ and $\mathrm{NAO}+(\mathrm{PNA}-/ \mathrm{NAO}+)$; average NAO index for active phase (Active); average NAO index for inactive phase (Inactive)

\begin{tabular}{lclllllc}
\hline & NAO $(\%)$ & PNA $(\%)$ & Corr & Mis $(\%)$ & PNA-/NAO+ $(\%)$ & Active & Inactive \\
\hline ECHAM4 & 11.78 & 6.40 & -0.37 & 63.9 & 32.1 & 0.29 & -0.27 \\
ECHAM5 & 9.62 & 6.66 & -0.22 & 57.8 & 28.5 & 0.18 \\
EGMAM & 11.14 & 5.17 & -0.41 & 65.6 & 34.1 & 0.17 \\
NCEP & 10.58 & 5.53 & 0.01 & 55.1 & 28.6 & -0.35 \\
ERA40 & 11.70 & 5.78 & 0.02 & 53.5 & 27.9 & 0.043 & 0.026 \\
\end{tabular}

For further details see text 
Fig. 1 Winter anomalies associated with the PNA index for the complete CGCMs runs. Compared are all winters with negative PNA index minus all winters with positive PNA index. Left column $500 \mathrm{hPa}$ geopotential height for a ECHAM4, c ECHAM5, and e EGMAM. Right column $500 \mathrm{hPa}$ storm track for b ECHAM4, d ECHAM5, and f EGMAM. Dashed lines indicate negative values, full lines positive values. Contour respectively. Significant differences at the 95\% (99\%) level are indicated by light (heavy) shading (red positive; blue negative) interval is $10 \mathrm{gpm}$ and $1 \mathrm{hPa}$,
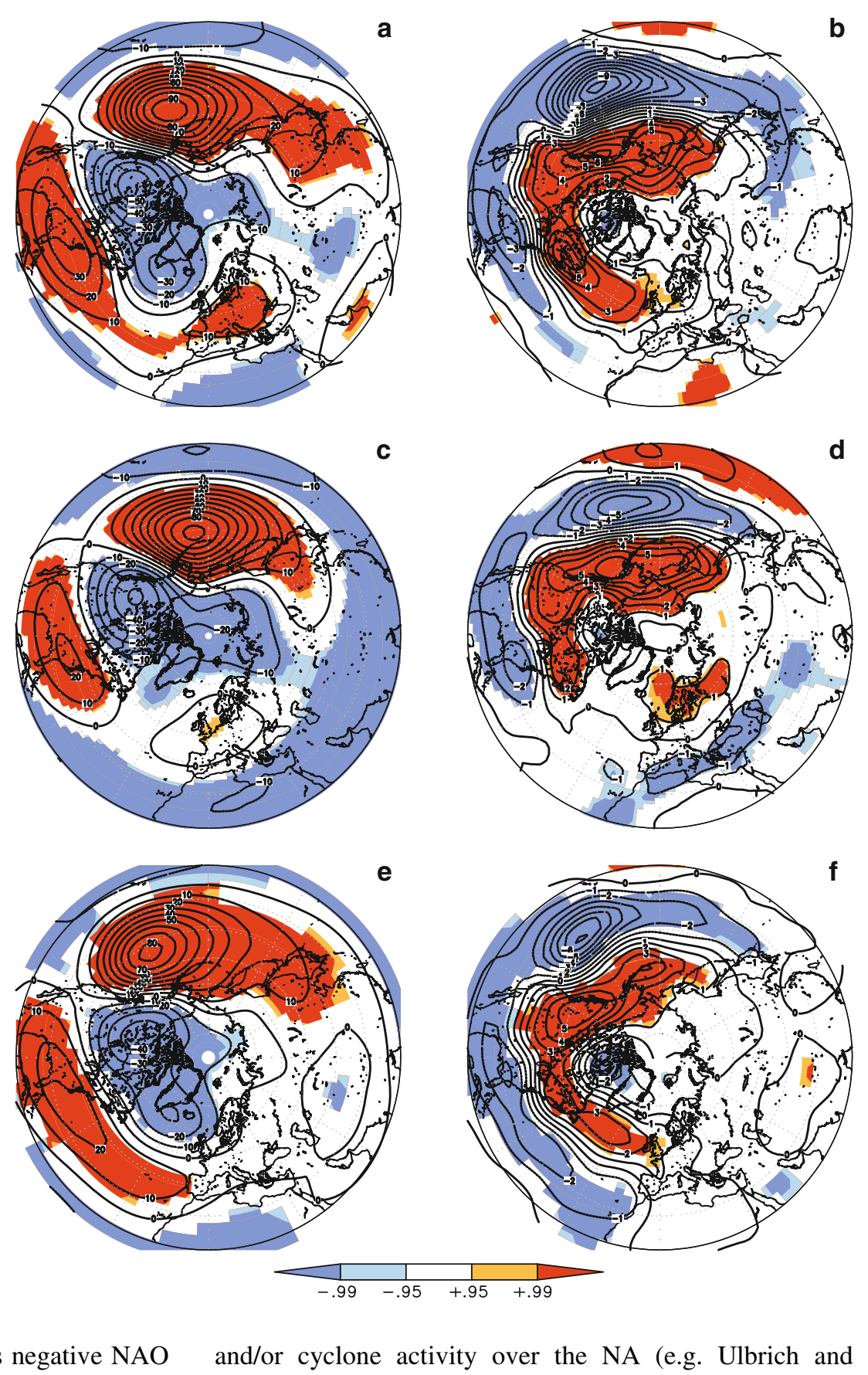

between winters with positive index versus negative NAO index). We focus now on results for ECHAM4 and ERA40, but the conclusions for the other datasets are similar. The impact of the NAO-phase is largest over the eastern NA (Fig. 3), with enhanced activity between Greenland and Northern Europe during positive NAO phases, and with reduced activity around $30^{\circ} \mathrm{N}-40^{\circ} \mathrm{N}$. In the area between Iceland and the British Isles, large percentages of storm track variability are explained by NAO (more than $50 \%$ in ECHAM4, circa $40 \%$ in ERA40 data, correlation fields not shown). Thus, the exit of the NA storm track is strongly coupled with NAO. This conclusion is in agreement with previous work on the link between NAO and storm track and/or cyclone activity over the NA (e.g. Ulbrich and Christoph 1999; Trigo et al. 2002; Raible 2007). On the other hand, the NAO influence on the intensity of the storm track maximum over Newfoundland is rather small (only about $10 \%$ explained variance, correlation field not shown) both in the CGCM and observations. It is thus smaller than the PNA influence (which corresponds to $30 \%$ explained variance for ECHAM4, cf. above) in the same region. In fact, the PNA influence remains significant at this location even if the NAO-related variability is removed (not shown). Hence, we conclude that the PNA influence on the NA storm track core over Newfoundland is a robust feature in the considered CGCM simulations. 
Fig. 2 Winter anomalies associated with the PNA index for the complete reanalysis data sets. Compared are all winters with negative PNA index minus all winters with positive PNA index. Left column $500 \mathrm{hPa}$ geopotential height for a NCEP, c ERA40. Right column $500 \mathrm{hPa}$ storm track for $\mathbf{b}$ NCEP, d ERA40. Dashed lines indicate negative values, full lines positive values. Contour interval is $10 \mathrm{gpm}$ and $1 \mathrm{hPa}$, respectively. Significant differences at the $95 \%$ (99\%) level are indicated by light (heavy) shading (red positive; blue negative)
Fig. $3500 \mathrm{hPa}$ storm track winter anomalies associated with the NAO index for a complete ECHAM4 run and b complete ERA40 data set. Compared are all winters with positive NAO index minus all winters with negative NAO index. Dashed lines indicate negative values, full lines positive values. Contour interval is $1 \mathrm{hPa}$. Significant differences at the $95 \%$ (99\%) level are indicated by light (heavy) shading (red positive; blue negative)
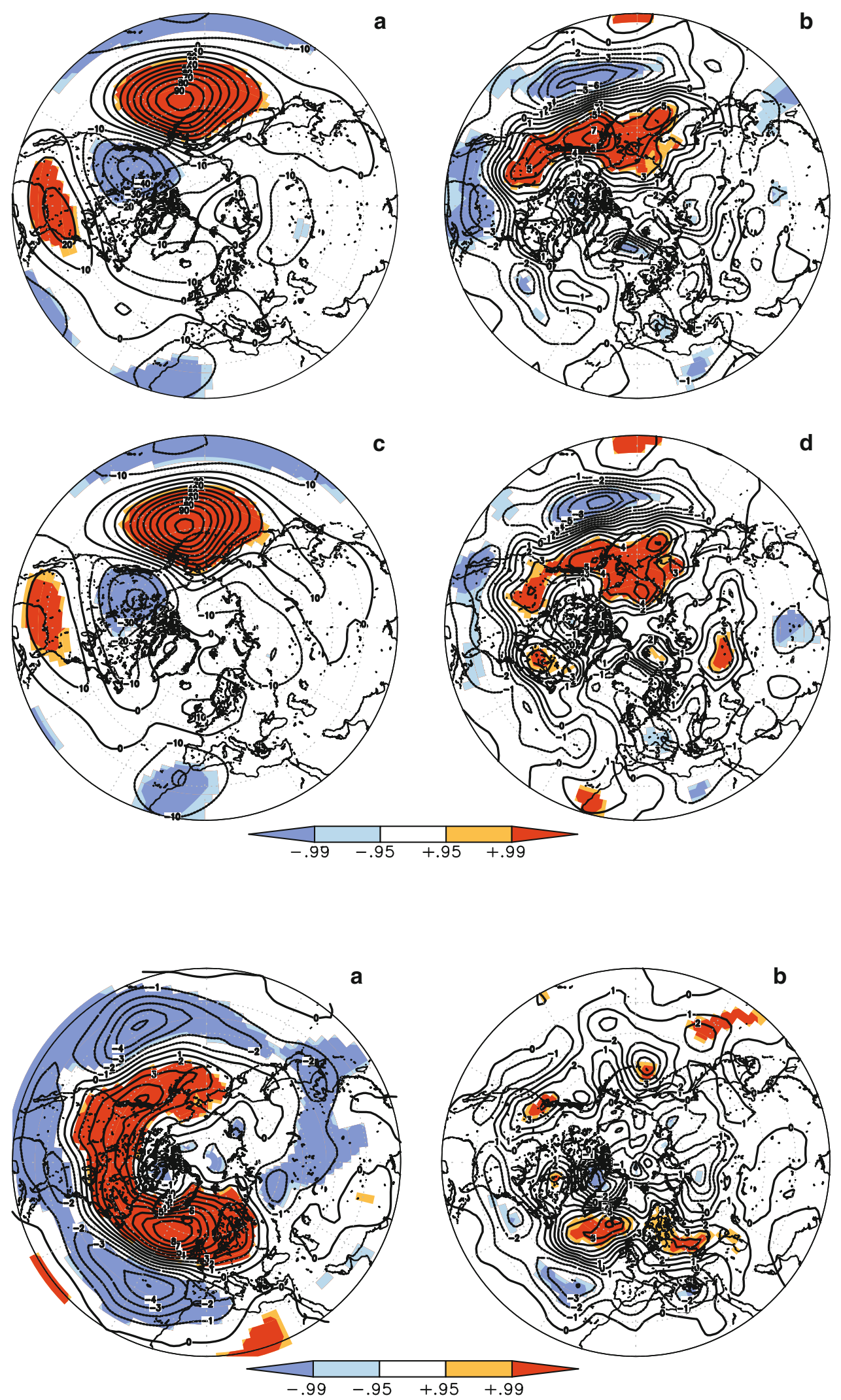
3.2 Decadal periods of weak and strong PNA-NAO relationships

Following the approach of running correlations also used by Raible et al. (2001), Honda et al. (2001) and Knippertz et al. (2003), we explore to what extent long term variations of the PNA-NAO coupling can be found in the CGCM runs. Figure 4 shows 21-year running mean correlations for the three CGCMs. Large low frequency variability of the coupling is found on decadal scales and longer time scales, so that time slices with PNA-NAO correlations similar to the observational PNA-NAO values (close to zero, cf. also Table 1) can be found in all three CGCM runs. Notably, the three CGCMs feature different characteristics of the running correlation time series. ECHAM4 has more long term variations than ECHAM5, but clearly less than what EGMAM produces. For each CGCM, we selected one period of strong anti-correlation (red boxes in Fig. 4) and one of weak correlation (blue boxes in Fig. 4) on the basis of the criterion that they lasted over at least 30 years. They are called "active" (corresponding to the terms used by Raible et al. 2001) if the coupling is strong and "inactive" if it is weak. Note that the chosen periods are not equal in length. We have performed several sensitivity studies focussing on shorter time windows to test the robustness of the results. Even though the storm track anomaly patterns do show small changes in location and intensity depending on the sub-period, the results of this analysis and in particular the mechanism (proposed later in Sect. 3.4) are still valid if one considers averaging over several active periods during one run or individual periods. The same is true if the chosen periods are slightly shifted.

A first check is performed with respect to the distributions of sign and intensity of the PNA and NAO winter indices in active and inactive periods. No evidence is found that active and inactive phases are different in this respect (see e.g. Fig. 5, from EGMAM model, in which the differences in correlations are particularly pronounced). This is also confirmed by comparing the index values' means and standard deviations between the two CGCMs.

The observational datasets are too short to identify active or inactive phases with the same rules as applied for the CGCMs. However, Honda et al. $(2001,2005)$ found two active periods, namely the 1973-1994 period, and the earlier (roughly) 1930-1950 period, separated by an inactive phase. Therefore, we followed Honda et al. (2001) and used a 21-year running mean correlation between the PNA and NAO indexes for NCEP (black curve) and ERA40 (grey curve) (Fig. 6). As expected (e.g. from Chang 2004, who investigated the correlation of storm tracks in the two basins), the correlation coefficient varies over time, even though it does not exceed a correlation of -0.3 (ERA40 between 1970 and 1990). This result also agrees with the
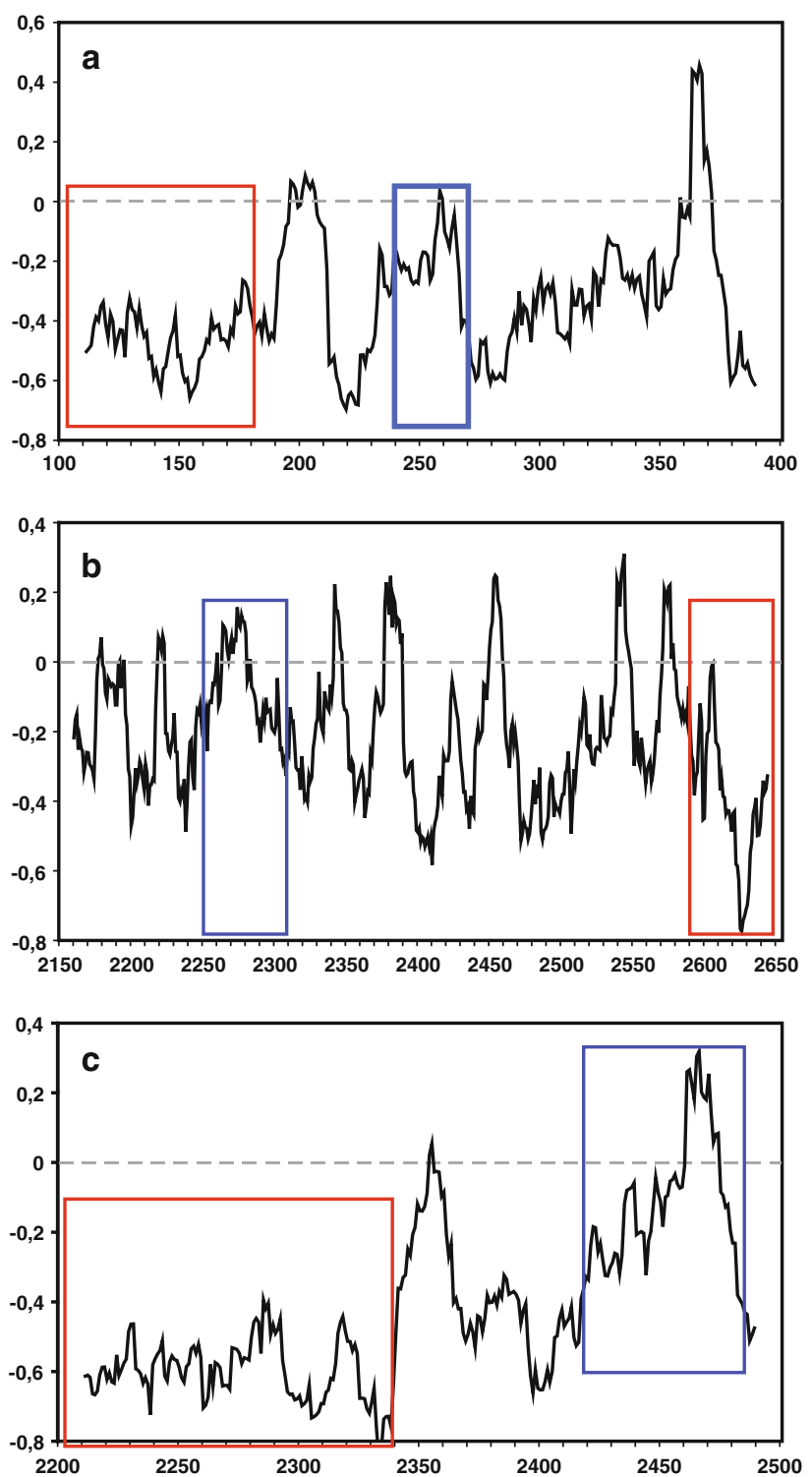

Fig. 4 21-year running mean PNA-NAO correlations of a ECHAM4, b ECHAM5, and c EGMAM. $X$-axis values correspond to model years. Active (inactive) periods with strong (weak) anticorrelation are indicated with red (blue) boxes. For further details see text

investigations of Honda et al. (2001), who identified 19731994 as a period of above normal coupling between the atmospheric circulation over the NA and the NP. The location of the time window corresponding to this period is marked with a red vertical line in Fig. 6. We also checked the distribution of PNA and NAO index values. In conjunction with the results for the CGCMs, we found no evidence of a distribution change between the active and inactive phase for the reanalysis datasets (not shown). The same is true for the mean intensities and spatial patterns of the storm tracks over the NP and the NA oceanic basins (not shown). 


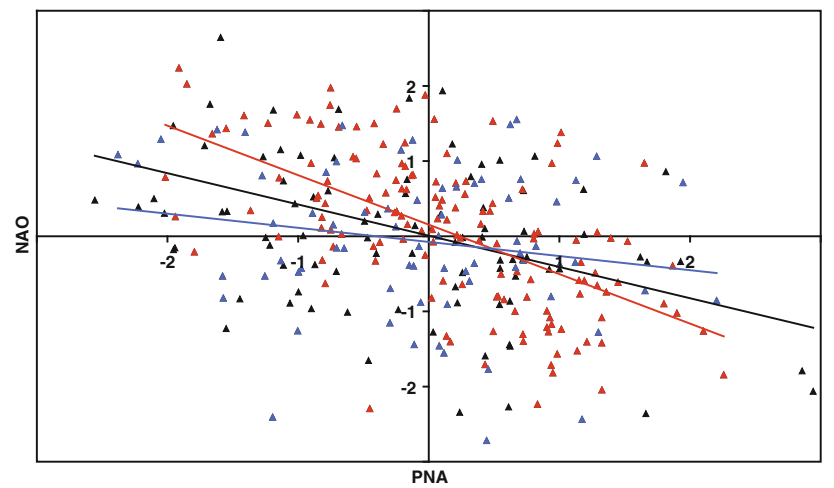

Fig. 5 Scatter plot for winter mean indices (November-March) of PNA and NAO for EGMAM. Red Dots active phase; blue dots inactive phase; black dots other years. Red line regression for the active period; blue line regression for the inactive period; black line regression for full 300 year control run. Active period: model years 2200-2340; inactive period: model years 2420-2490 (cf. red and blue boxes in Fig. 4c)

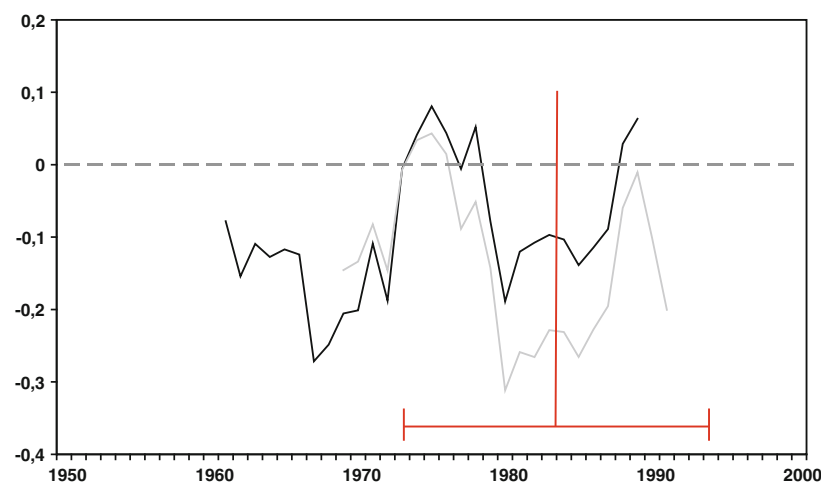

Fig. 6 21-year running mean PNA-NAO correlations of NCEP (black line) and ERA40 (grey line) for historical years. The red line corresponds to the 21-year running mean spanning 1973-1994

\subsection{PNA-NAO relationships in active versus inactive phases}

The question of the characteristic difference between active and inactive phases is now addressed comparing composites for the PNA effects (PNA- minus PNA+) computed for the active and inactive periods. CGCM results shown focus on those obtained from ECHAM4 as an example, but results for the other models are also discussed (cf. below). With respect to $500 \mathrm{hPa}$ geopotential height fields, anomalies over the centres of the PNA pattern are larger for the active period (Fig. 7a) than for the inactive period (Fig. 7c; note that the different size of the coloured areas of statistical significance is also influenced by the different lengths of the active versus the inactive period). The individual PNA centres are, however, not equally affected in the different CGCMs: For the ECHAM 5, the positive $500 \mathrm{hPa}$ geopotential height anomaly over Florida (as part of the
PNA pattern) becomes very weak during the inactive phase (not shown), while the effect is less pronounced in the other two models (Fig. 7c, EGMAM not shown). With respect to the NP storm track, anomaly maxima over the NP (associated with the PNA) are located over the Aleutian Islands in the inactive phase (Fig. 7d), while they are found in the vicinity of the North American coastline in the active phase (Fig. 7b). The differences between active and inactive phase with respect to the storm tracks are larger over the North American continent and over NA storm track area. For the former, the influence of the PNA becomes very small during the inactive phase (Fig. 7d). During the active phase, there is a very strong influence of PNA on the NA storm track core and downstream over the NA, indicating enhanced storm track activity for PNA- (red areas in Fig. 7b). The same is valid for EGMAM and ECHAM5, which also show strong storm track anomalies over Newfoundland for PNA- in the active phase, even if such anomalies are comparatively smaller than those located in the NP storm track area (not shown). In contrast, only a weak positive anomaly close to Florida (i.e., south of the climatological storm track maximum) is associated with PNA+, both for active and inactive phases (blue areas in Fig. 7b, d). Again, the results for the other two CGCMs are similar.

Distinguishing the period 1973-1994 (active) from the complete period in the observational datasets, the respective composites for ERA40 and NCEP give similar results. During the active period, PNA influence on the $500 \mathrm{hPa}$ geopotential height centres is increased (Fig. 8a, c) compared to complete periods (Fig. 2), particularly in the area near Florida. For the NA storm track, a large area with positive anomalies extends from Newfoundland (now significant also for NCEP data) to Iberia in the active phase (Fig. 8b, d). Please note that the significances are weak in Fig. 8, even though the anomalies are larger than in Fig. 2. This is primarily due to the small number of years used (only 21).

\subsection{Possible reasons for the variable link between PNA and NAO}

In this section, we search for a potential role of the storm track in the variable connection between PNA and NAO. Several concurrent and inter-related mechanisms associated with the growth conditions for cyclones may influence wave activity over the eastern NA, which is eventually exerting a barotropic feedback on the mean flow associated with the NAO (e.g., Lau 1988; Lau and Nath 1991; Orlanski 1998). Figure 9 shows a scheme of a typical situation with positive NAO and negative PNA. These situations are associated with merging subpolar centres of the NAO and the Canadian centre of the PNA, suggesting 
Fig. 7 Winter anomalies associated with the PNA index for different periods in the ECHAM4 run. Compared are winters with negative PNA index minus winters with positive PNA index. Left column $500 \mathrm{hPa}$ geopotential height for a active phase, $\mathbf{c}$ inactive phase. Right column $500 \mathrm{hPa}$ storm track for $\mathbf{b}$ active phase, $\mathbf{d}$ inactive phase. Periods: Active phase: model years 100 180; inactive phase: model years 240-270 (cf red and blue boxes in Fig. 4a). Dashed lines indicate negative values, full lines positive values. Contour interval is $10 \mathrm{gpm}$ and $1 \mathrm{hPa}$, respectively. Significant differences at the $95 \%(99 \%)$ level are indicated by light (heavy) shading (red positive; blue negative)
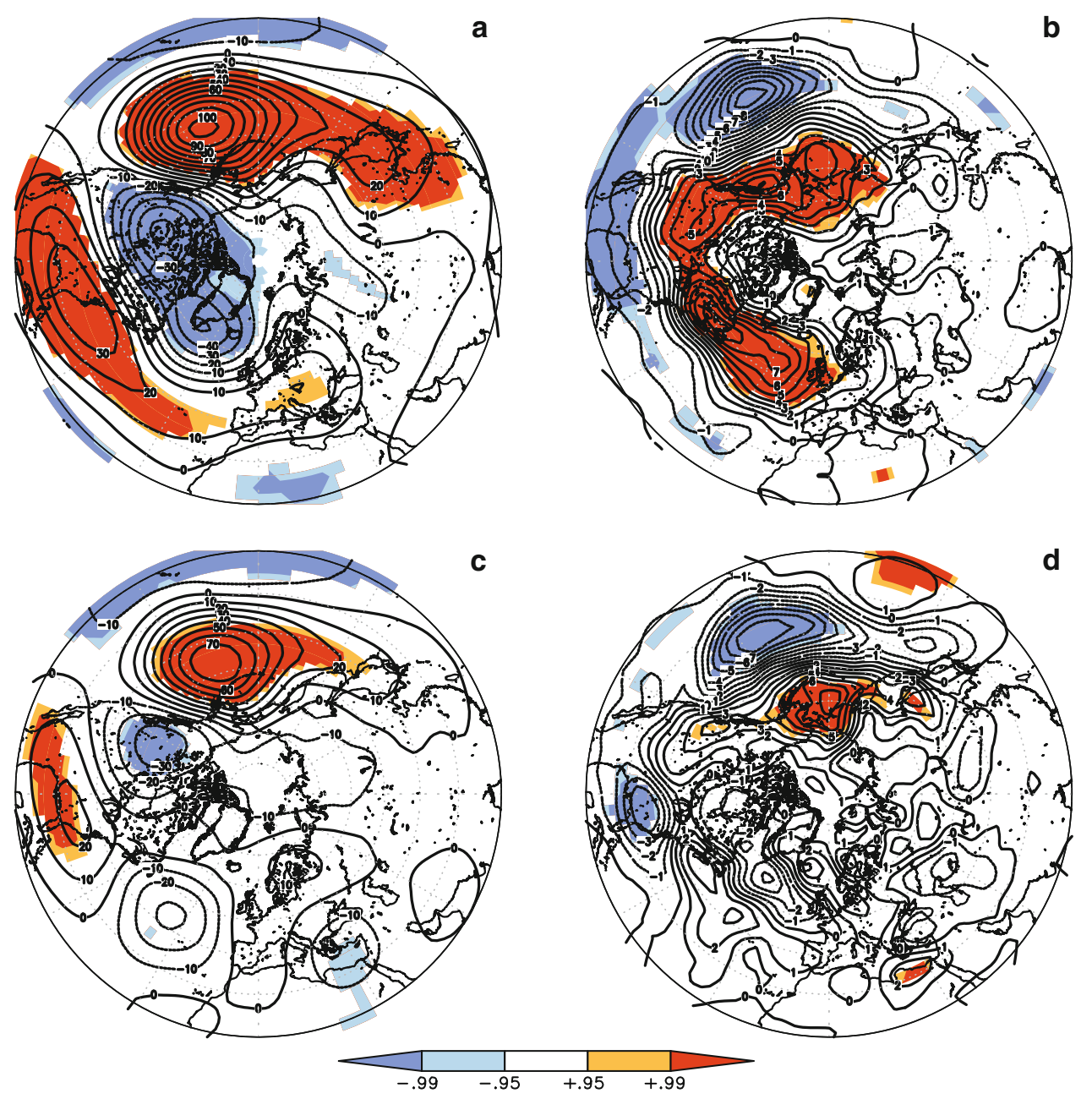

that eddies may move almost zonally from the Pacific to the Atlantic, so that their amplitudes are larger when they enter the baroclinic zone over the eastern North American continent. These growth conditions themselves are modified with the phases of NAO and PNA, as during the active phase (Fig. 9), the low pressure anomaly over Canada leads to an advection of cold and dry air from the Arctic in the direction of the Eastern North American Coast. On the other hand, the anomalous high pressure in the subtropics (associated with the south-eastern pole of the PNA located close to Florida) leads to an advection of moist and warm air into the same area. The PNA effects on humidity and Eady growth rate as a measure of baroclinicity are shown for the complete ECHAM4 and NCEP data sets in Fig. 10a-d. During negative PNA, humidity is apparently advected northeastwards from the Gulf of Mexico towards the NA storm track core area (cf. Fig. 10a), so that eddies may use this latent heat for their growth, thereby increasing the NA storm track's intensity during the active phase. At the same time, baroclinicity is enhanced over the North American continent upstream of the NA storm track's maximum over Newfoundland (Fig. 10c, d). During positive PNA phases, both storm track enhancing factors are reduced accordingly. With respect to baroclinicity variability over this area, PNA explains more than $60 \%$ in the CGCMs, and $30-40 \%$ in the reanalyses (correlation fields not shown). The modulation of this factor is in turn associated with corresponding changes of polar jet intensity over the eastern North America and the western NA (Fig. 10e, f).

The NAO itself also modifies the storm track over Newfoundland (Fig. 3) as well as upstream humidity, baroclinicity and jet stream. Even after removing the NAO influence by linear regression, the above results concerning the influence of PNA on NA storm track growth conditions remain largely valid (cf. Sect. 3.1). Still, PNA and NAO are not always in opposite phases (Table 1). There must be an additional mechanism eventually modulating the NAO response. Following Honda and Nakamura (2001) who suggested an effect of the seasonal cycle on the link of the Aleutian and Icelandic low through baroclinic activity, we search for such a mechanism analysing the storm track anomalies for single winter months, looking into the full ECHAM4 run as an example: for the PNA- phase, 
Fig. 8 Winter anomalies associated with the PNA index for 1973-1994 in the reanalysis data sets. Compared are winters with negative PNA index minus winters with positive PNA index. Left column $500 \mathrm{hPa}$ geopotential height for a NCEP, c ERA40. Right column $500 \mathrm{hPa}$ storm track for b NCEP, d ERA40. Dashed lines indicate negative values, full lines positive values. Contour interval is $10 \mathrm{gpm}$ and $1 \mathrm{hPa}$, respectively. Significant differences at the 95\% (99\%) level are indicated by light (heavy) shading (red positive; blue negative)
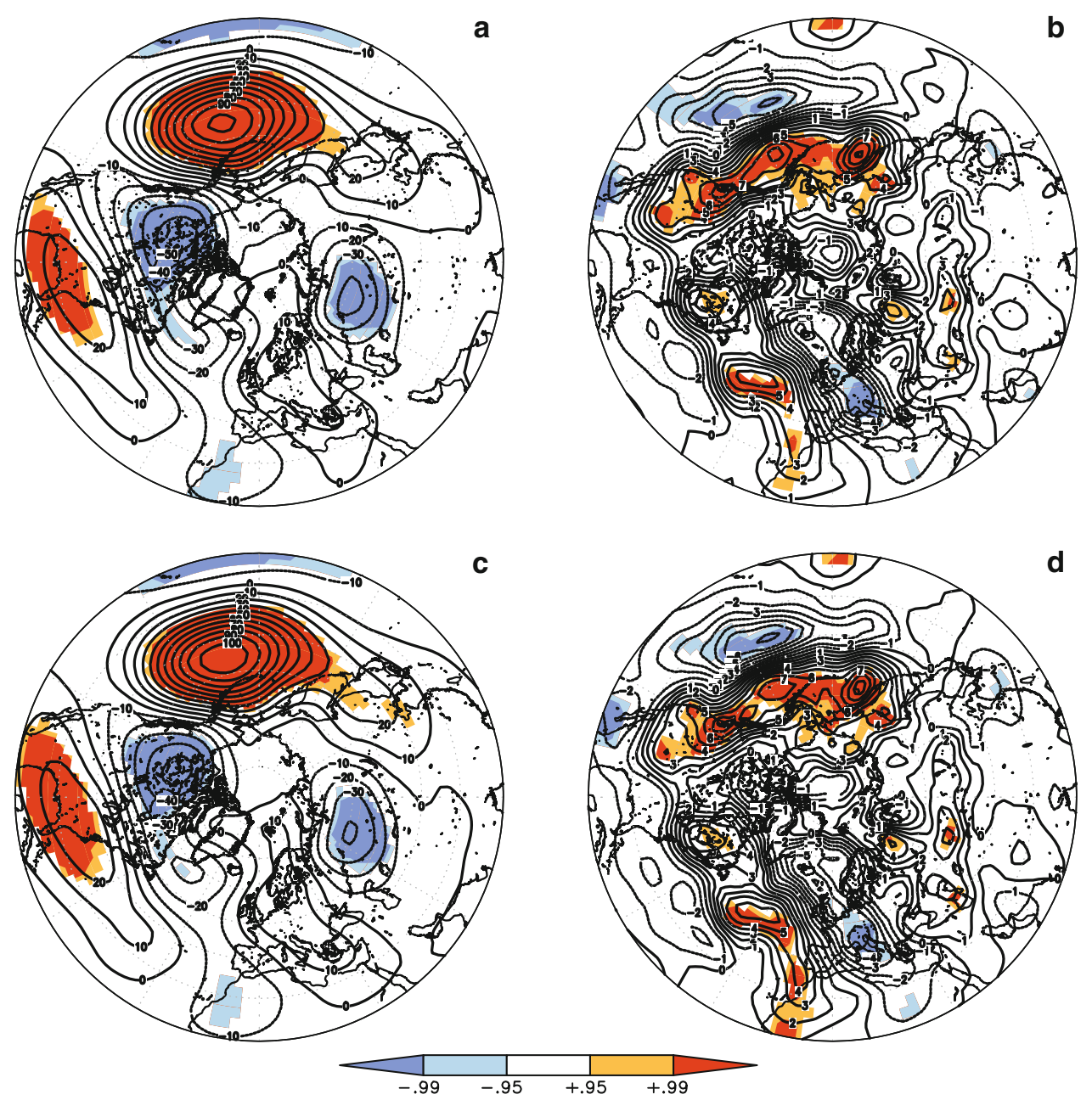

Fig. 9 General conditions for negative PNA and simultaneously positive NAO denoting the different influences to the storm track core. " $H$ " and " $L$ " represents areas with positive and negative geopotential height anomalies, respectively. For further details see text

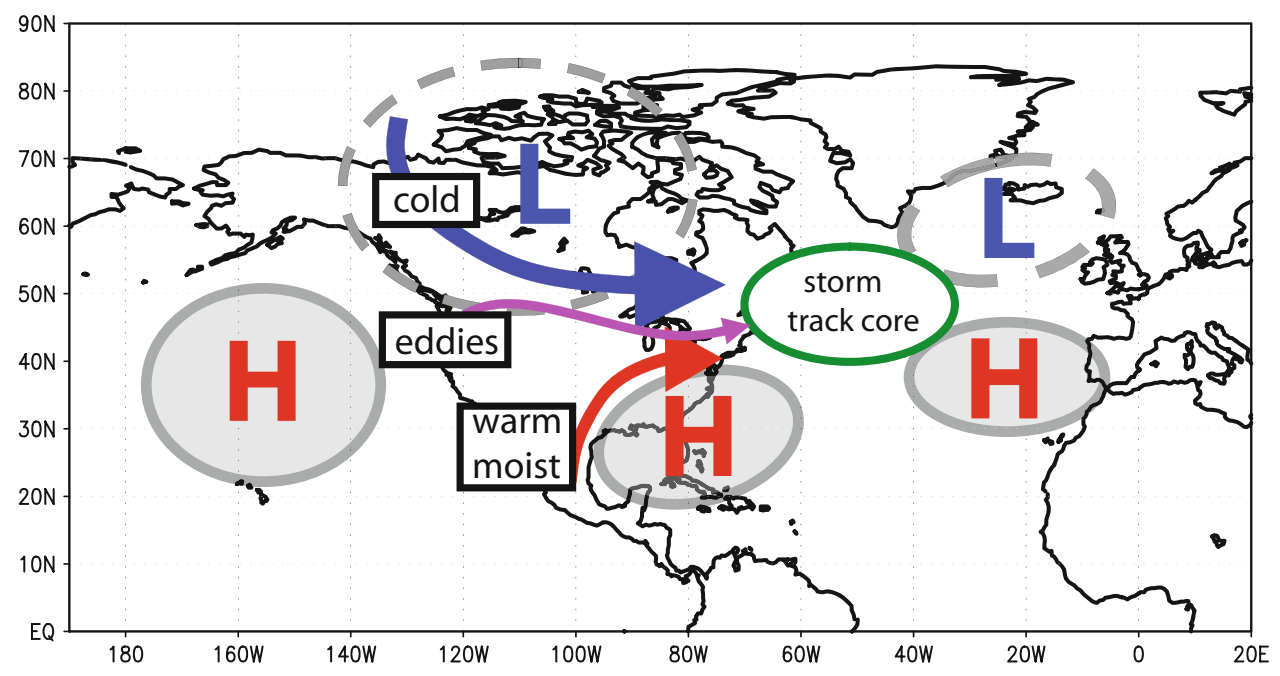

positive storm track anomalies over the Newfoundland area are already observed for November (Fig. 11a). They intensify in December and January (Fig. 11b, c) and extend downstream to Europe during February and March (Fig. 11d, e). For the PNA+ phase, enhanced storm track anomalies near Florida (blue values in Fig. 11) are strongest between November and January, weakening afterwards. This suggests that on average there is a strong PNA influence in early winter, shifting the NA storm track core area. The extended downstream influence in late winter 
Fig. 10 Winter anomalies associated with the PNA index for the complete ECHAM4 run and NCEP data set. Compared are winters with negative PNA index minus winters with positive PNA index. Left column anomalies for ECHAM4 for a $850 \mathrm{hPa}$ humidity, c $400 \mathrm{hPa}$ Eady growth rate, $\mathbf{e}$ $250 \mathrm{hPa}$ jet stream. Right column anomalies for NCEP for b $850 \mathrm{hPa}$ humidity, d $400 \mathrm{hPa}$ Eady growth rate, f $250 \mathrm{hPa}$ jet stream. Dashed lines indicate negative values, full lines positive values. Contour interval is $1 \times 10^{-4} \mathrm{~kg} / \mathrm{kg}, 1 \times 10^{-2}$ day $^{-1}$ and $1 \mathrm{~m} / \mathrm{s}$, respectively. Significant differences at the 95\% (99\%) level are indicated by light (heavy) shading (red positive; blue negative)
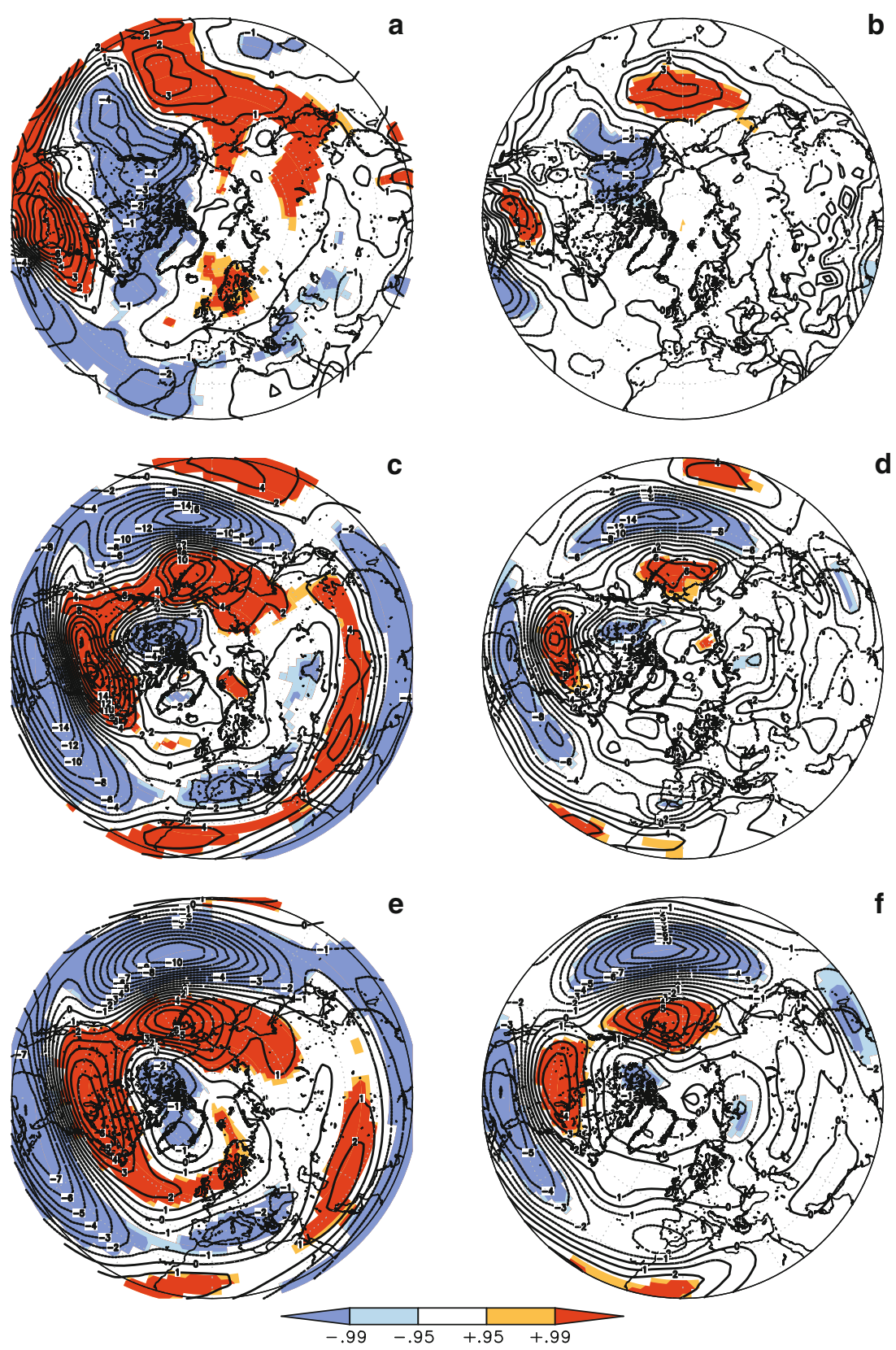

could indicate a change of the NAO to a positive phase under the influence of PNA-. In particular, the results of Fig. 11 also document the stability of the PNA signal in the NA storm track core area over the whole winter.

Turning back to the distinction between the active and inactive phase, there should be a difference in how the conditions enhancing the storm track over Newfoundland (thus supporting the establishment of positive NAO for negative PNA phases) and those not supporting develop seasonally during the winter. Based on ECHAM4 results, Fig. 12 provides evidence of the validity of this hypothesis. Positive storm track anomalies over Newfoundland increase from November to January in active phase winters (Fig. 12a, c), while no such development is not found for the inactive phase (Fig. 12b, d). The monthly development of moisture advection and Eady growth rate anomalies from November to March for the active phase shows a corresponding development of moisture advection and Eady growth rate anomalies, which increase from November towards a peak in January, still being visible until March. During the passive phase, the anomalies are much weaker and decrease sharply already in February. Note that areas with significant differences are smaller in when considering just the active and passive phases in 
Fig. 11 Seasonal development of $500 \mathrm{hPa}$ storm track monthly anomalies associated with the PNA index for the complete ECHAM4 run. Compared are months with negative PNA index minus months with positive PNA index. a November, b December, c January, d February, e March. Dashed lines indicate negative values, full lines positive values. Contour interval is $2 \mathrm{hPa}$. Significant differences at the 95\% (99\%) level are indicated by light (heavy) shading (red positive; blue negative)
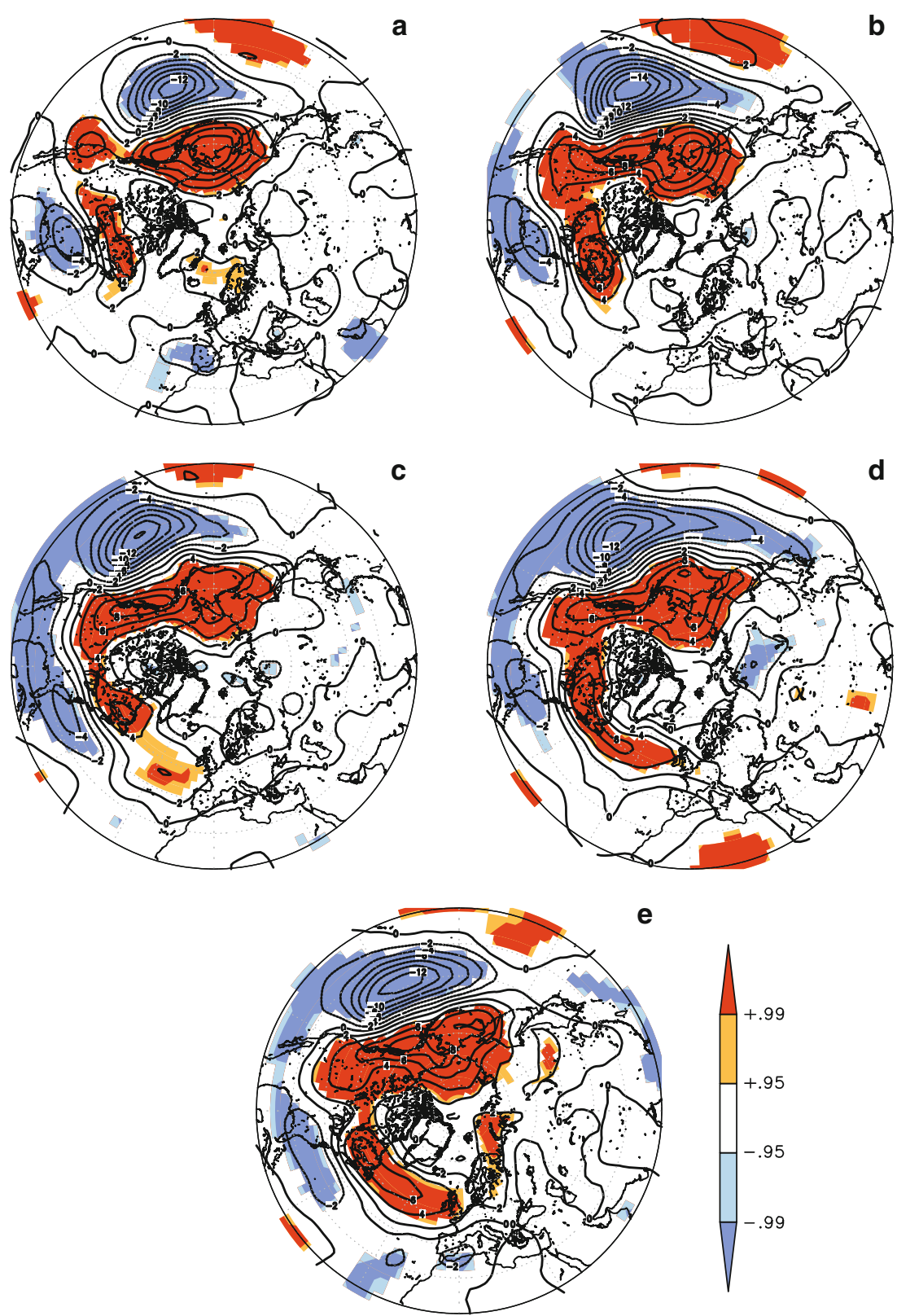

Fig. 12 compared to the results for whole run shown in Fig. 11. This is due to the reduced number of years used for the sub-periods.

From the assumption of a direct effect of the storm track on the NAO, the NAO index values should be typically larger during negative PNA phases than during positive PNA phases. This is indeed the case in all five datasets we have analysed (cf. last 2 columns in Table 1). Of course, the discussed link between PNA and NAO is only explaining part of NAO decadal variability. As mentioned before, the NAO itself produces conditions favourable (in the positive phase) to the intensification of the Newfoundland storm track core, contributing to the variability of the system.

\section{Discussion and concluding remarks}

We considered three multi-century CGCM simulations which closely reproduce the observed NAO and PNA patterns with respect to location and variability. Further, we focussed our considerations of the troposphere, as two of the three CGCMs considered do not include a well resolved stratosphere. A significant negative correlation between the winter means of PNA and NAO indexes was found in all three CGCM runs. Composite fields for winters with negative versus positive PNA suggest a physical background of the correlations which can be summarized as follows: PNA modifies growth conditions of baroclinic waves, which can exert a strong influence on the NA storm track core over 
Fig. 12 Seasonal development of $500 \mathrm{hPa}$ storm track monthly anomalies associated with the PNA index for different periods in the ECHAM4 run. Compared are months with negative PNA index minus months with positive PNA index. Left column anomalies for the active phase for a November, c January. Right column anomalies for the inactive phase for b November, d January. Periods: Active phase: model years 100-180; inactive phase: model years 240-270 (cf. red and blue boxes in Fig. 4a). Dashed lines indicate negative values, full lines positive values. Contour interval is $2 \mathrm{hPa}$. Significant differences at the 95\% (99\%) level are indicated by light (heavy) shading (red positive; blue negative)
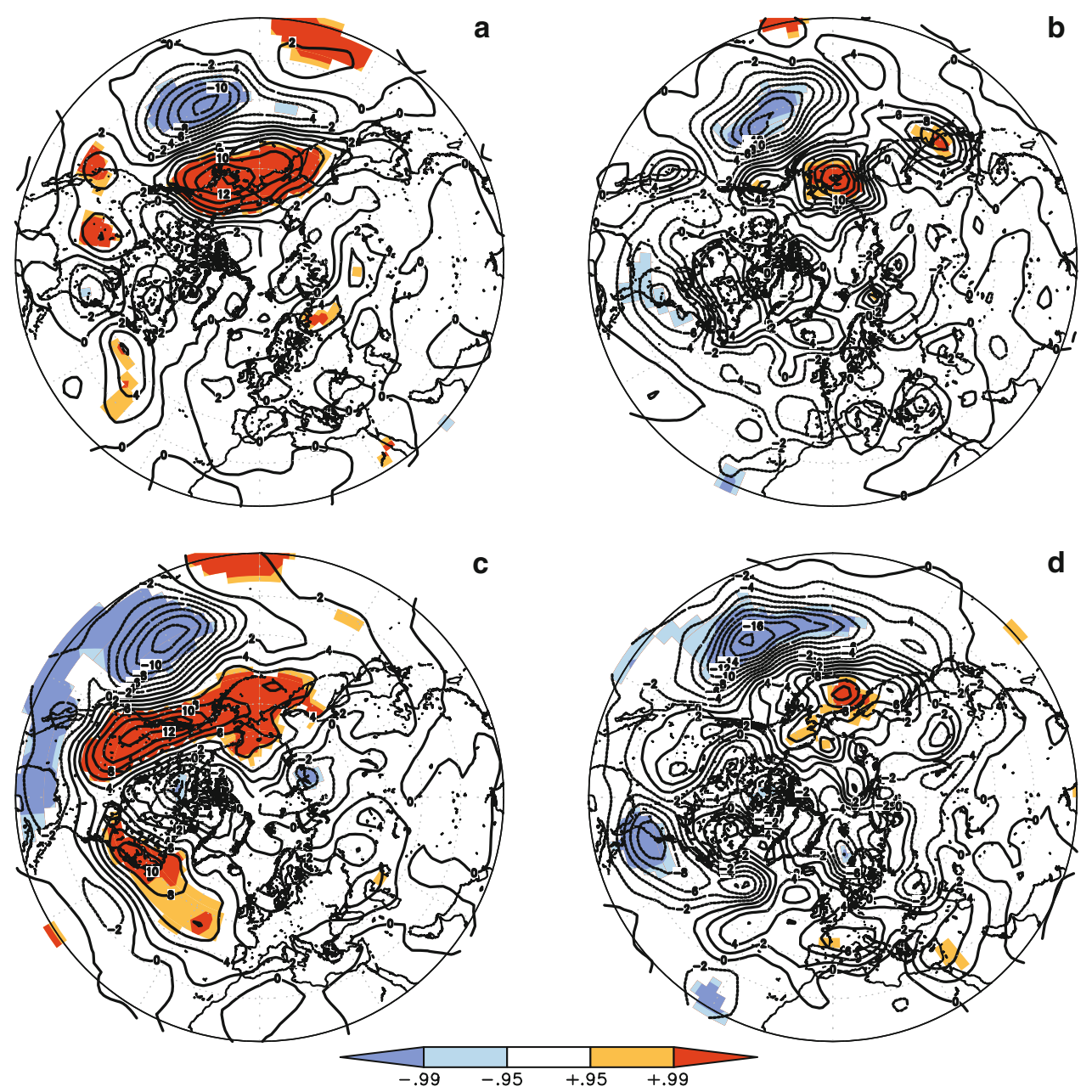

c. During negative PNA and positive NAO phases, enhanced baroclinic wave activity is found in a band reaching from the NP to the NA. Eddies moving along this path may enter the (climatological) baroclinic zone upstream of Newfoundland with larger initial amplitude, so that their amplitudes are eventually increased over Newfoundland (pink arrow in Fig. 9). During positive PNA phases, eddies at this location are reduced.

Eddies in the NA storm track will impose a barotropic feedback on the NAO. This part of the chain has not been explicitly considered in this paper, as the basic process was confirmed in other studies (e.g., Orlanski 1998; Orlanski and Gross 2000). Basically, enhanced eddy activity would lead to reduced SLP to the north and rising SLP to the south. In addition to the PNA induced eddy variations and feedbacks, there is also an "internal" NAO-eddy feedback. However, the NAO's influence on the storm track is largest over the eastern NA, while over Newfoundland, PNA influence is larger than NAO influence (cf. Sect. 3.1, Figs. 1, 2, 3). Based on the seasonal development of the storm track anomalies, we investigated how the PNA may influence the NAO phase 
according to CGCM data. Results show an enhanced Newfoundland storm track maximum in the early winter for PNA - and a subsequent enhancement of the storm track over the whole NA in the subsequent months. As the storm track over the central and eastern Atlantic is closely related to the NAO variability, this development can be explained by the shift of the NAO index to more positive values.

Beyond the rather modest (though significant) correlations of PNA and NAO in the CGCMs, periods of strong and weak coupling ("active" and "inactive phases") can be identified by applying moving correlations. From this point of view, the whole reanalysis period could correspond on average to a weaker coupling phase in the CGCMs, as correlation- and composite patterns in the CGCMs and Reanalysis are similar. Nevertheless, phases with stronger/ weaker coupling can also be identified in the reanalysis period (in accordance with Honda et al. 2001, 2005). Our results go well with conclusions from studies focussing Aleutian-Icelandic Low Seesaw, which modulates of the storm tracks over the Caribbean/Western Atlantic (Honda et al. 2001, 2005; Honda and Nakamura 2001). In particular, Honda and Nakamura (2001) identified a seasonal evolution of the Aleutian-Icelandic Low Seesaw, with a late winter peak, which is consistent with our findings about the seasonal evolution of the storm track modulation (Fig. 10). This suggests that the mechanisms found in the models are potentially also connecting PNA and NAO pattern (and the Aleutian and Icelandic lows) in the real world.

We have not tried to address differences between the three CGCMs in our study, in spite of different mean anticorrelations (Table 1) and the differences with respect to decadal variations of the coupling intensity. In particular, it would be interesting to explore the importance of the stratosphere in a next step, which is well represented in the EGMAM model (cf. Hübener et al. 2007; Spangehl et al. 2009). From our study, no first order effect is visible. Baldwin and Dunkerton $(1999,2001)$ suggested that large circulation anomalies in the observed lower stratosphere are related to substantial shifts in the AO/NAO phases, as large positive values of the AO/NAO indexes are much more probable for strong stratospheric vortex regimes. One may speculate that such a mechanism leads to the stronger anti-correlation of PNA and NAO in EGMAM compared to the other models.

Our study was restricted to the investigation of the link between PNA and NAO as two atmospheric modes. It is well known that PNA is closely related to ENSO variability (e.g. Straus and Shukla 2002), and the link between ENSO and NAO or European climate has been addressed in several studies (e.g. Pozo-Vázquez et al. 2005). Further work should address the nature of this link, taking into account the decadal variability of the PNA-NAO connection discussed here. Additionally, we will address the question of maintenance of the coupling from one winter to the next in the active phase, and the regime transition to an inactive period, which was not investigated in the present study.

Acknowledgments We would like to thank Erich Roeckner and the MPI for Meteorology (Hamburg, Germany) for support and the ECHAM4 and ECHAM5 data, and Ulrich Cubasch for the EGMAM data. We thank DKRZ/WDCC (Hamburg, Germany) and ZAIK/ RRZK (Cologne, Germany) for computer and storage capacity. We are indebted to Michael Christoph (Univ. Cologne, Germany), Thomas Spangehl and Falk Niehörster (both FU Berlin, Germany) for fruitful discussions. We thank two anonymous reviewers for their comments that helped to improve the manuscript.

Open Access This article is distributed under the terms of the Creative Commons Attribution Noncommercial License which permits any noncommercial use, distribution, and reproduction in any medium, provided the original author(s) and source are credited.

\section{Appendix}

See Figs. 13, 14, and 15.
Fig. 13 Climatological $500 \mathrm{hPa}$ storm track winter for a complete ECHAM4 run and b complete ERA40 data set. Values are given in gpm, isolines each $5 \mathrm{gpm}$
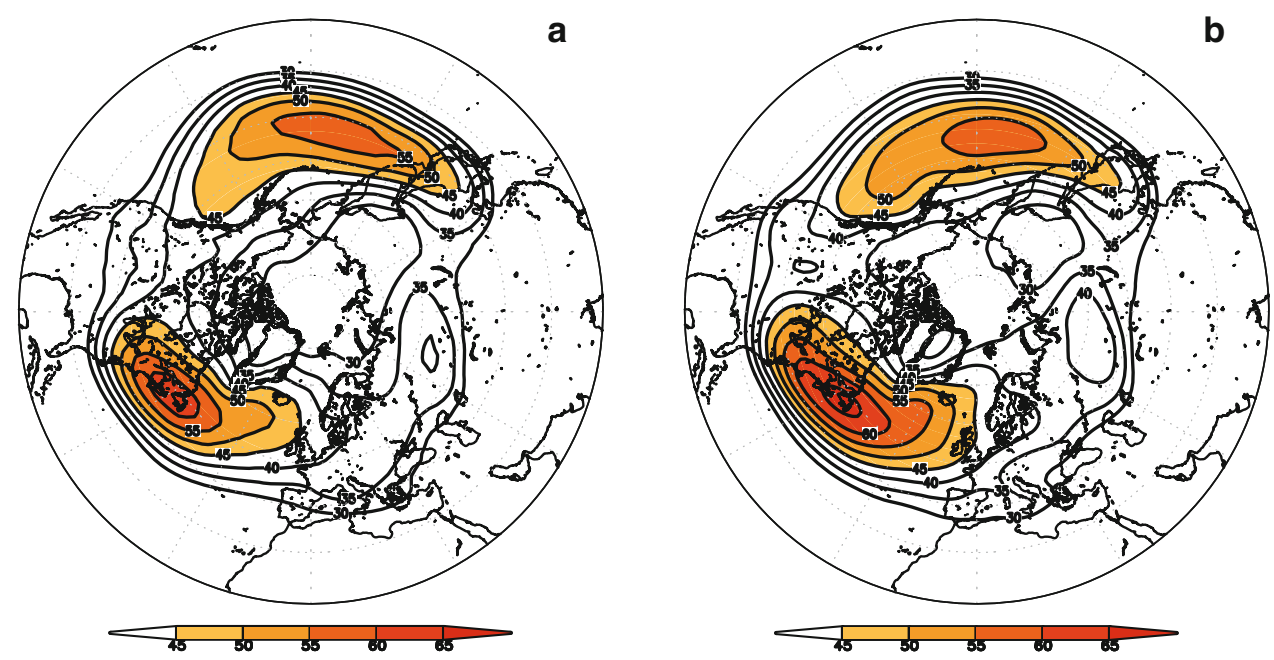
Fig. 14 NAO and PNA patterns obtained as rotated EOF's for the CGCM data sets. Left column NAO patterns for a ECHAM4, c ECHAM5, and e EGMAM. Right column PNA patterns for b ECHAM4, d ECHAM5, and f EGMAM. Periods: ECHAM4: 300-year control run; ECHAM5: 505year control run; EGMAM: 300-year control run
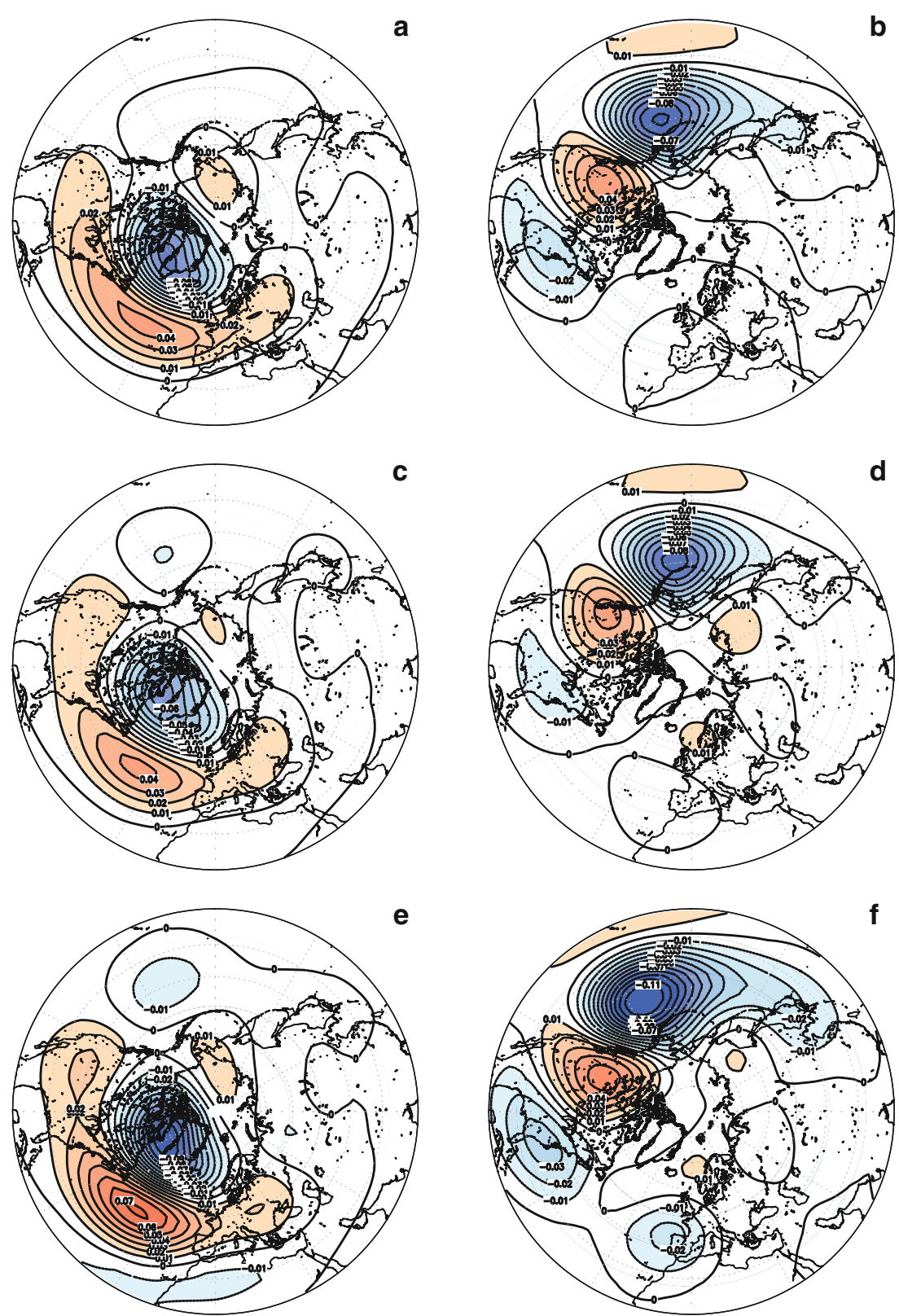
Fig. 15 NAO and PNA patterns obtained as rotated EOF's for the reanalysis data sets. Left column NAO patterns for a NCEP, c ERA40. Right column PNA patterns for $\mathbf{b}$ NCEP, $\mathbf{d}$ ERA40. Periods: NCEP: 19501999; ERA40: 1958-2001
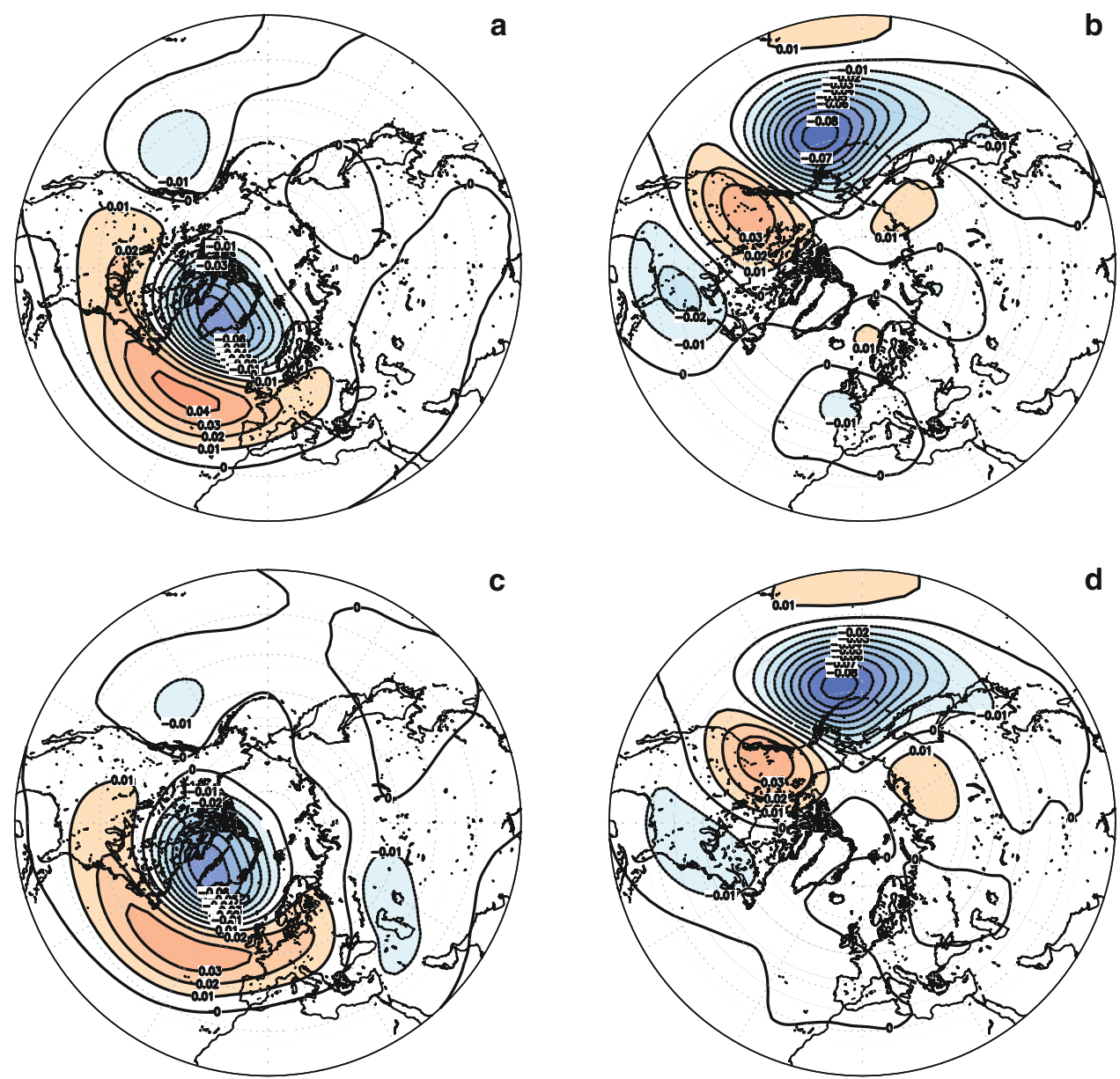

\section{References}

Ambaum MHP, Hoskins BJ (2002) The NAO troposphere-stratosphere connection. J Clim 15:1969-1978

Archambault HM, Bosart LF, Keyser D, Aiyyer AR (2008) Influence of large-scale flow regimes on cool-season precipitation in the northeastern United States. Mon Weather Rev 136:2945-2963

Bacher M, Oberhuber JM, Roeckner E (1998) ENSO dynamics and seasonal cycle in the tropical Pacific as simulated by the ECHAM4/OPYC3 coupled general circulation model. Clim Dyn $14: 431-450$

Baldwin MP, Dunkerton TJ (1999) Propagation of the Arctic Oscillation from the stratosphere to the troposphere. J Geophys Res 104:30937-30946

Baldwin MP, Dunkerton TJ (2001) Stratospheric harbingers of anomalous weather regimes. Science 294:581-584

Barnston AG, Livezey RE (1987) Classification, seasonality and persistence of low-frequency atmospheric circulation patterns. Mon Weather Rev 115:1083-1126

Benedict J, Lee S, Feldstein SB (2004) Synoptic view of the North Atlantic Oscillation. J Atmos Sci 61:121-144

Bengtsson L, Hodges KI, Roeckner E, Brokopf R (2006) On the natural variability of the pre-industrial European climate. Clim Dyn 27:743-760

Blackmon ML (1976) A climatological spectral study of the $500 \mathrm{mb}$ geopotential height of the Northern Hemisphere. J Atmos Sci 33:1607-1623

Bongioannini Cerlini P, Corti S, Tibaldi S (1999) An intercomparison between low-frequency variability indices. Tellus A 51:773-789
Castanheira JM, Graf HF (2003) North Pacific-North Atlantic relationships under stratospheric control? J Geophys Res 108:4036. doi:10.1029/2002JD002754

Castanheira JM, Liberato MLR, De La Torre L, Graf HF, DaCamara CC (2009) Baroclinic Rossby wave forcing and Barotropic Rossby wave response to stratospheric vortex variability. J Atmos Sci 66:902-914. doi:10.1175/2008JAS2862.1

Chang EKM (2004) Are the Northern Hemisphere winter storm tracks significantly correlated? J Clim 17:4230-4244

Christoph M, Ulbrich U, Haak U (1995) Faster determination of the intraseasonal variability of storm tracks using Murakami's recursive filter. Mon Weather Rev 123:578-581

Christoph M, Ulbrich U, Oberhuber JM, Roeckner E (2000) The role of ocean dynamics for low-frequency fluctuations of the NAO in a coupled ocean-atmosphere GCM. J Clim 13:25362549

Croci-Maspoli M, Schwierz C, Davies HC (2007) Atmospheric blocking: space-time links to the NAO and PNA. Clim Dyn 29:713-725

Feldstein SB (2002) Fundamental mechanisms of the growth and decay of the PNA teleconnection pattern. Quart J R Meteorol Soc 128:775-796

Feldstein SB (2003) The dynamics of NAO teleconnection pattern growth and decay. Quart J R Meteorol Soc 129:901-924

Franzke C, Lee S, Feldstein SB (2004) Is the North Atlantic Oscillation a breaking wave? J Atmos Sci 61:145-160

Franzke C, Feldstein SB, Lee S (2009) Synoptic analysis of the Pacific-North American teleconnection pattern. Quart J R Meteorol Soc (submitted) 
Grötzner A, Latif M, Barnett TP (1998) A decadal climate cycle in the North Atlantic Ocean as simulated by the ECHO coupled GCM. J Clim 11:831-847

Hoerling MP, Hurrell JW, Xu T (2001) Tropical origins for recent North Atlantic climate change. Science 292:90-92

Honda M, Nakamura H (2001) Interannual seesaw between the Aleutian and Icelandic lows. Part II: its significance in the interannual variability over the wintertime northern hemisphere. J Clim 14:4512-4529

Honda M, Nakamura H, Ukita J, Kousaka I, Takeuchi K (2001) Interannual seesaw between the Aleutian and Icelandic lows. Part I: seasonal dependence and life cycle. J Clim 14:1029-1042

Honda M, Yamane S, Nakamura H (2005) Impacts of the AleutianIcelandic Low Seesaw on surface climate during the twentieth century. J Clim 18:2793-2802

Hoskins BJ, Hodges KI (2002) New perspectives on the Northern Hemisphere winter storm tracks. J Atmos Sci 59:1041-1061

Hoskins BJ, Valdes PJ (1990) On the existence of storm-tracks. J Atmos Sci 47:1854-1864

Hübener H, Cubasch U, Langematz U, Spangehl T, Niehörster F, Fast I, Kunze M (2007) Ensemble climate simulations using a fully coupled ocean-troposphere-stratosphere GCM. Phil Trans R Soc Ser A 365:2089-2101

Hurrell JW (1995) Transient eddy forcing of the rotational flow during northern winter. J Atmos Sci 52:2286-2301

Hurrell JW, Kushnir Y, Visbeck M, Ottersen G (2003) An overview of the North Atlantic Oscillation. In: Hurrell JW, Kushnir Y, Ottersen G, Visbeck M (eds) The North Atlantic Oscillation: climate significance and environmental impact. Geophys Monogr Ser, vol 134, pp 1-35

Jungclaus JH, Botzet M, Haak H, Keenlyside N, Luo JJ, Latif M, Marotzke J, Mikolajewicz U, Roeckner E (2006) Ocean circulation and tropical variability in the coupled model ECHAM5/MPI-OM. J Clim 19:3952-3972

Kistler R et al (2001) The NCEP/NCAR 50-year reanalysis: monthlymeans CD-ROM and documentation. Bull Am Meteorol Soc 82:247-267 co-authors

Knippertz P, Wernli H (2010) A Lagrangian climatology of tropical moisture exports to the Northern Hemispheric extratropics. J Clim 23:987-1003. doi: 10.1175/2009JCLI3333.1

Knippertz P, Ulbrich U, Marques F, Corte-Real J (2003) Decadal changes in the link El Niño, NAO and European/North African rainfall. Int J Climatol 23:1293-1311

Lau NC (1988) Variability of the observed midlatitude storm tracks in relation to low-frequency changes in the circulation pattern. J Atmos Sci 45:2718-2743

Lau NC, Nath MJ (1991) Variability of the baroclinic and barotropic transient eddy forcing associated with monthly changes in the midlatitude storm tracks. J Atmos Sci 48:2589-2613

Lin H, Derome J, Brunet G (2005) Tropical Pacific link to the two dominant patterns of atmospheric variability. Geophys Res Lett 32:L03801. doi:10.1029/2004GL021495

Luo D, Gong T, Diao Y (2007) Dynamics of eddy-driven low frequency dipole modes. Part III: meridional displacement of westerly jet anomalies during two phases of NAO. J Atmos Sci 64:3232-3248

Manganello JV (2008) The influence of sea surface temperature anomalies on low-frequency variability of the North Atlantic Oscillation. Clim Dyn 30:621-641

Manzini E, McFarlane NA (1998) The effect of varying the source spectrum of a gravity wave parameterization in a middle atmosphere general circulation model. J Geophys Res 103:31523-31539

Min SK, Legutke S, Hense A, Kwon WT (2005) Internal variability in a $1000-y r$ control simulation with the coupled climate model
ECHO-G-II. El Niño Southern Oscillation and North Atlantic Oscillation. Tellus A 57:622-640

Müller WA, Roeckner E (2008) ENSO teleconnections in projections of future climate in ECHAM5/MPI-OM. Clim Dyn 31:533-549

Müller WA, Frankignoul C, Chouaib N (2008) Observed decadal tropical Pacific-North Atlantic teleconnections. Geophys Res Lett 35:L24810. doi:10.1029/2008GL035901

Orlanski I (1998) Poleward deflection of storm tracks. J Atmos Sci 55:2577-2602

Orlanski I, Gross B (2000) The life cycle of baroclinic eddies in a storm track environment. J Atmos Sci 57:3498-3513

Orsolini YJ, Kvamstø NG, Kindem IT, Honda M, Nakamura H (2008) Influence of the Aleutian-Icelandic Low Seesaw and ENSO onto the stratosphere in ensemble winter hindcasts. J Meteorol Soc Jpn 86:817-825

Pinto JG, Ulbrich U, Leckebusch GC, Spangehl T, Reyers M, Zacharias S (2007) Changes in storm track and cyclone activity in three SRES ensemble experiments with the ECHAM5/MPIOM1 GCM. Clim Dyn 29:195-210

Pinto JG, Zacharias S, Fink AH, Leckebusch GC, Ulbrich U (2009) Factors contributing to the development of extreme North Atlantic cyclones and their relationship with the NAO. Clim Dyn 32:711-737

Pozo-Vázquez D, Gámiz-Fortis SR, Tovar-Pescador J, Esteban-Parra MJ, Castro-Díez Y (2005) North Atlantic Winter SLP anomalies based on the autumn ENSO state. J Clim 18:97-103

Raible CC (2007) On the relation between extremes of midlatitude cyclones and the atmospheric circulation using ERA40. Geophys Res Lett 34:L07703. doi:10.1029/2006GL029084

Raible CC, Luksch U, Fraedrich K, Voss R (2001) North Atlantic decadal regimes in a coupled GCM simulation. Clim Dyn 18:321-330

Rivière G, Orlanski I (2007) Characteristics of the Atlantic stormtrack eddy activity and its relation with the North Atlantic Oscillation. J Atmos Sci 64:241-266

Rodwell MJ, Rowell DP, Folland CK (1999) Oceanic forcing of the wintertime North Atlantic Oscillation and European climate. Nature 398:320-323

Roeckner E et al (2003) The atmospheric general circulation model ECHAM 5. PART I: model description. MPI Report 349

Roeckner E, Oberhuber JM, Bacher A, Christoph M, Kirchner I (1996) ENSO variability and atmospheric response in a global coupled atmosphere-ocean GCM. Clim Dyn 12:737-754

Roeckner E, Brokopf R, Esch M, Giorgetta M, Hagemann, Kornblueh L, Manzini E, Schlese U, Schulzweida U (2006) Sensitivity of simulated climate to horizontal and vertical resolution. J Clim 19:3771-3791

Rogers JC, Raphael MN (1992) Meridional eddy sensible heat fluxes in the atmosphere in the extremes of the Pacific/North American teleconnection pattern. J Clim 5:127-139

Saito K, Cohen J (2003) The potential role of snow cover in forcing interannual variability of the major Northern Hemisphere mode. Geophys Res Lett 30:1302. doi:10.1029/2002GL016341

Scaife AA, Knight JR, Vallis GK, Folland CK (2005) A stratospheric influence on the winter NAO and North Atlantic surface climate. Geophys Res Lett 32:L18715. doi:10.1029/2005GL023226

Schneidereit A, Blender R, Fraedrich K, Lunkheit F (2007) Iceland climate and North Atlantic cyclones in ERA40 reanalyses. Meteorol Z 16:17-23

Song J, Li C, Zhou W, Pan J (2009) The linkage between the PacificNorth American teleconnection pattern and the North Atlantic Oscillation. Adv Atmos Sci 26:229-239

Spangehl T, Cubasch U, Raible CC, Langematz U, Schimanke S, Körper J, Hofer D (2009) Transient climate simulations from the maunder minimum to present day: the role of the stratosphere. 
J Geophys Res-CAWSES special issue. doi:10.1029/ 2009JD012358

Sterl A, van Oldenborgh GJ, Hazeleger W, Burgers G (2008) On the robustness of ENSO teleconnections. Clim Dyn 29:469-485

Straus DM, Shukla JS (2002) Does ENSO force the PNA? J Clim 15:2340-2358

Thompson DWJ, Wallace JM (1998) The arctic oscillation signature in the wintertime geopotential height and temperature fields. Geophys Res Lett 25:1297-1300

Thompson DWJ, Wallace JM (2000) Annular modes in the extratropical circulation. Part I: month-to-month variability. J Clim 13:1000-1016

Thompson DWJ, Baldwin MP, Wallace JM (2002) Stratospheric connection to Northern Hemisphere wintertime weather: implications for predictions. J Clim 15:1421-1428

Timmermann A, Latif M, Voss R, Grötzner A (1998) North Atlantic interdecadal variability: a coupled air-sea mode. J Clim 11:1906-1931

Trenberth KE, Branstator GW, Karoly D, Kumar A, Lau NC, Ropelewski C (1998) Progress during TOGA in understanding and modeling global teleconnections associated with tropical sea surface temperatures. J Geophys Res 103:14291-14324

Trigo RM, Osborn TJ, Corte-Real J (2002) The North Atlantic Oscillation influence on Europe: climate impacts and associated physical mechanisms. Clim Res 20:9-17
Ulbrich U, Christoph M (1999) A shift of the NAO and increasing storm track activity over Europe due to anthropogenic greenhouse gas forcing. Clim Dyn 15:551-559

Uppala SM et al (2005) The ERA-40 reanalysis. Quart J R Meteorol Soc 131:2961-3012

van Loon H, Rogers JC (1978) The seesaw in winter temperatures between Greenland and Northern Europe. Part I: general description. Mon Weather Rev 106:296-310

Legutke S, Voss R (1999) The Hamburg atmosphere-ocean coupled climate circulation model ECHO-G. DKRZ Technical report nr. 18, Deutsches Klimarechenzentrum, Hamburg, Germany

Wallace JM (2000) North Atlantic Oscillation/annular mode: two paradigms-one phenomenon. Quart J R Meteor Soc 126:791-805

Wallace JM, Gutzler DS (1981) Teleconnections in the geopotential height field during the Northern Hemisphere winter. Mon Weather Rev 109:784-812

Wanner H, Bronnimann S, Casty C, Gyalistras D, Luterbacher J, Schmutz C, Stephenson DB, Xoplaki E (2001) North Atlantic Oscillation: concepts and studies. Surv Geophys 22:321-382

Woollings TJ, Hoskins BJ, Blackburn M, Berrisford P (2008) A new Rossby wave-breaking interpretation of the North Atlantic Oscillation. J Atmos Sci 65:609-626 